clinical baseline and a wider neck independently predict an increased risk of neurological morbidity/mortality.

Disclosures A. Griffin: None. E. Hauck: None.

\section{E-012 PREDICTORS OF ANEURYSM RECURRENCE AFTER ENDOVASCULAR EMBOLIZATION WITH THE SMART COIL SYSTEM}

M Anadani ${ }^{*}$, M Sattur, E Almallouhi, S Al kasab, A Spiotta. Neurology, Medical University of South Carolina, Charleston, SC

\subsection{6/neurintsurg-2019-SNIS.87}

Background/Objective Endovascular aneurysm embolization is effective and safe. However, the angiographic recurrence continues to be an issue after endovascular embolization. In this study, we aimed to report the rate of recurrence at 1-year follow up angiography and to investigate the predictors of recurrence.

Methods We used the SMART registry to identify subgroup of patients who underwent endovascular embolization with SMART coils and had one year follow up angiography. The primary outcome of this study was angiography recurrence at 1 year. We used stepwise regression analysis to identify the predictors of recurrence.

Results A total of 466 patients were included of whom 78 $(16 \%)$ had recurrence at 1 year follow up angiography. Mean age was 60 years and 74\% were female. $112(24 \%)$ of treated aneurysms were ruptured and $125(27 \%)$ were $\geq 4 \mathrm{~mm}$ in size. On Multivariate analysis, Immediate post procedure Raymond II and III (vs Raymond I), neck size $\geq 4$ (vs. $<4$ ), stentassisted coiling, and age were associated with the risk of recurrence at one-year follow-up.

Conclusion In this study we identified the predictors of aneurysm recurrence after endovascular embolization with SMART coils.

Disclosures M. Anadani: None. M. Sattur: None. E. Almallouhi: None. S. Al kasab: None. A. Spiotta: None.

\section{E-013 DELAYED POST-OPERATIVE COIL MIGRATION AFTER SUCCESSFUL BALLOON-ASSISTED COILING OF A BASILAR APEX ANEURYSM}

S Ahmed*, J Mann, L Peeling, M Kelly. Neurosurgery, University of Saskatchewan, Saskatoon, SK, Canada

10.1136/neurintsurg-2019-SNIS.88

A 69-year-old female patient presented with a ruptured small basilar apex aneurysm. Balloon-assisted coil embolization was performed, and the patient remained stable post-operatively. A CT-scan performed 12 days after the procedure showed coil migration from the aneurysm, and repeat angiography confirmed coil migration into the left proximal posterior cerebral artery segment. The patient gave consent for a revision procedure, where an LVIS Jr. stent and 7 micro-coils were placed into the aneurysm to re-secure the aneurysm. The patient suffered no further complications and was discharged home on dual antiplatelet therapy. Modified Rankin Score at 90-days was 1 . We review the literature regarding delayed coil migration, and discuss management considerations.

Disclosures S. Ahmed: None. J. Mann: None. L. Peeling: None. M. Kelly: None.

\section{$\mathrm{E}-014$ \\ COIL EMBOLIZATION RESULTS OF THE RUPTURED PROXIMAL POSTERIORI INFERIOR CEREBELLAR ARTERY ANEURYSM: A SINGLE-CENTER 10 YEARS' EXPERIENCE}

${ }^{1} \mathrm{Y}$ Jung ${ }^{*},{ }^{1} \mathrm{H}$ Lee, ${ }^{1} \mathrm{~J}$ Kim, ${ }^{2} \mathrm{C}$ Chang. ${ }^{1}$ Neurovascular surgery/Neurointervention, Daegu, Korea, republic of; ${ }^{2}$ Professor, Daegu, Korea, republic of

\subsection{6/neurintsurg-2019-SNIS.89}

Objective To report a single-center experience with endovascular treatment of ruptured proximal posterior inferior cerebellar artery (PICA) aneurysms.

Methods Between January 2007 and December 2016, among 1403 patients with aneurysmal subarachnoid hemorrhage, 15 with ruptured proximal PICA aneurysms underwent endovascular embolization at our institution. Aneurysmal obliteration with a single microcatheter was performed in 9 patients. Additional microcatheter or stent-assisted coil embolization was performed in 4 patients and parent artery occlusion in 2 patients.

Results Immediate angiographic results showed 10 complete occlusions $(66.7 \%, 10 / 15)$. Five patients showed incomplete occlusion (remnant neck in 4 patients, remnant aneurysm in 1). Of those, 2 patients experienced recurrence and required conversion to microsurgical clipping. The remaining 2 patients remained in relatively stable condition. Procedure-related complications occurred in 3 patients (20\%, with thromboembolic complications in 2 patients and intraprocedural rupture in 1). Clinical outcome was excellent: Glasgow Outcome Score 4 or 5 in 12 of 15 patients (80\%). There was no rebleeding during follow-up.

Conclusions Ruptured proximal PICA aneurysms may be effectively treated with endovascular coil embolization. A variety of coil embolization techniques are required to obliterate an aneurysm without parent artery occlusion. Given that recurrence is possible, follow-up is required. Surgical clipping can be performed for recurrence with a relatively low risk of complications, because the aneurysm is unruptured. Coil embolization of a proximal PICA aneurysm in the acute phase can be a good treatment modality with good patient outcomes.

Disclosures Y. Jung: None. H. Lee: None. J. Kim: None. C. Chang: None.

\section{E-015 INFLAMMATION IN MURINE ANEURYSM HEALING: THE ROLE OF CXCL1}

D Patel ${ }^{*}$, K Motwani, K Hosaka, B Hoh. Department of Neurosurgery, University of Florida Gainesville, FL

\subsection{6/neurintsurg-2019-SNIS.90}

Introduction Cerebral aneurysms affect up to 5\% of the U.S. population and can have devastating consequences. Rupture of cerebral aneurysms results in subarachnoid hemorrhage which has a mortality of up to $40 \%$. Current prophylactic treatment options include surgical placement of a clip or use of coils or flow diverters through an endovascular approach. These options carry significant complication risks with functional disability or death occurring in up to $17.5 \%$ of patients after surgical clipping and in up to $8.4 \%$ after endovascular treatment. A significant drawback of endovascular coiling is that up to $20 \%$ of aneurysms can recur and require retreatment due to incomplete thrombus formation and insufficient fibrotic healing. This high rate of recurrence warrants investigation 